\title{
Lugano Classification Advanced Stage Adult Hodgkin Lymphoma AJCC v8
}

National Cancer Institute

\section{Source}

National Cancer Institute. Lugano Classification Advanced Stage Adult Hodgkin

Lymphoma A/CC v8. NCI Thesaurus. Code C141196.

Advanced stage adult Hodgkin lymphoma based on the Lugano classification criteria. It includes stages III and IV. (from AJCC 8th Ed.) 